\title{
$(94) \%(2), 696-5$ \\ propiedades y aplicaciones de los materiales aislantes a base de vidrio espumado
}

\author{
CARLOS SANCHEZ CASTRO
}

\begin{abstract}
RESUMEN
En un artículo precedente, aparecido en el núm. 82 de U. A. M. C., se definieron y estudiaron brevemente los materiales aislantes a base de vidrio espumado -Foamglas-, dedicándose una atención más amplia a los procedimientos y ensayos de laboratorio, realizados para obtener la máxima espumación del producto. Entonces sólo se mencionaron algunas de sus características y propiedades más importantes: también se indicaron, sin entrar en detalles, algunas de sus aplicaciones. Seguidamente, y como complemento de lo anteriormente dicho, se van a enumerar: las caracteristicas de este material, pesos específicos, coefteientes de aislamiento, resistencias, etc.; los productos que con él se fabriçan, bloques, planchas, coquilias, etc, ; y las diștintas aplicaciones que tiene en la construcción $y$ en otras industrias, a las cuales beneficia con su colaboración, resolviendo problemas de aislamiento, aligeramiento de peso, etc. En distintas fotografías se presentan algunos ejemplos de aislamiento, de edificios, fijación de piezas y enlucido o revestimiento de los paramentos realizados con este producto. I
\end{abstract}

\section{CARACTERISTICAS}

El vidrio espumado Foamglas se viene empleando con fines constructivos e industriales desde el año 1940. Este material ha sido sometido a pruebas, con resultados satisfactorios, por organismos oficiales y particulares de los Estados Unidos. Sus excepcionales propiedades físicas y químicas han dado lugar a que sean muy numerosas sus aplicaciones en el campo de los aislamientos, dentro, claro está, del régimen adecuado de temperaturas que puede resistir satisfactoriamente.

Cada día es mayor la necesidad de emplear en la construcción materiales aislantes ligeros, cuyas características permanezcan inalterables a través del tiempo. El vidrio espumado es el material ideal desde dichos puntos de vista (ligereza, aislamiento e inalterabilidad). De todos es conocida la resistencia que presenta el cristal a los agentes químicos. Sólo existen contados productos que lo atacan o deterioran. Los materiales inorgáricos, como es sabido, son generalmente más inalterables que los orgánicos y no sufren ni la descomposición ni el ataque por los parásitos que experimentan los materiales procedentes, directa o indirectamente de los seres vivos. Aprovechando esta inalterabllidad y dotando al vidrio ordinario de aquellas características, de aislamiento, térmico y acústico, y ligereza, de que carecía, se ha llegado a un producto cuyas interesantes propiedades se indicarán a continuación. Estos datos se han obtenido de la abundante literatura comercial, publicada por la Pittsburgh Corning Corporation, fabricante del Foamglas. 


\section{COMPORTAMIENTO MECANICO}

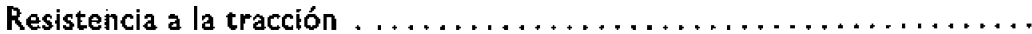

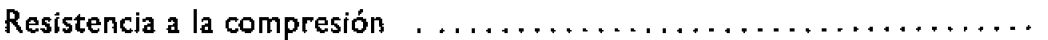

Resistencia a la flexión..............................

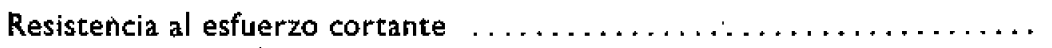

Módulo de elasticidad.

$\begin{array}{rr}5,9 & \mathrm{~kg} / \mathrm{cm}^{2} \\ 9,8 & \mathrm{~kg} / \mathrm{cm}^{2} \\ 7,03 & \mathrm{~kg} / \mathrm{cm}^{2} \\ 4,5 & \mathrm{~kg} / \mathrm{cm}^{2} \\ 14.061,0 & \mathrm{~kg} / \mathrm{cm}^{2}\end{array}$

\section{CARACTERISTICAS FISICAS}

Coeficiente de dilatación

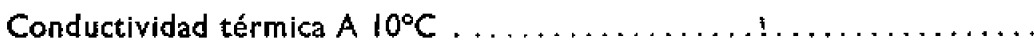

Conductividad térmica A $149^{\circ} \mathrm{C} \ldots \ldots \ldots \ldots \ldots \ldots \ldots \ldots \ldots \ldots$

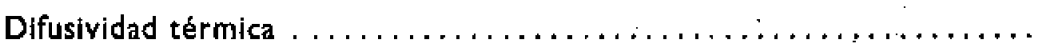

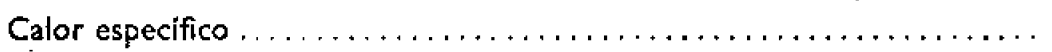

Densidad.

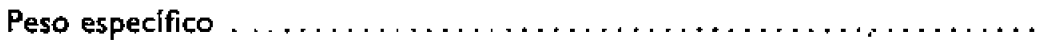

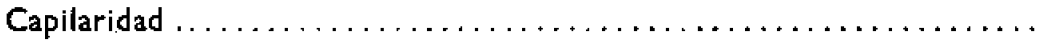

Absorción de agua $\ldots \ldots \ldots \ldots \ldots \ldots \ldots \ldots \ldots \ldots \ldots \ldots \ldots \ldots \ldots \ldots \ldots \ldots \ldots$

Higroscopicidad

$0,0000072 /^{\circ} \mathrm{C}$.

$0,0496 \mathrm{kcal} / \mathrm{m}^{2} / \mathrm{h} /{ }^{\circ} \mathrm{C} / \mathrm{mm}$

$0,0682 \mathrm{kcal} / \mathrm{m}^{2} / \mathrm{h} /{ }^{\circ} \mathrm{C} / \mathrm{mm}$

$0,0371 \mathrm{~m}^{2}$ por dla

$0,1111 \mathrm{kcal} / \mathrm{kg}$

$144,17 \mathrm{~kg} / \mathrm{m}^{3}$
0,144

0

$0.2 \%$ en volumen (realizado el ensayo con bloques de $50,8 \times 304,8 \times 457,2 \mathrm{~mm})$. no aumenta de peso después de permanecer 246 dias en un ambiente cuya humedad relativa es del $90 \%$.

\section{COMPORTAMIENTO ACUSTICO}

Aislamiento del sonido (ensayo realizado con una placa de Foamglas de $50,8 \mathrm{~mm}$ de espesor, sin revestir). Absorción del sonido

Coeficiente de reducción del sonido sin valor significativo

Transmisión del sonido. 0,12

factor medio de reducción del sonido, 28,3 decibelios.

\section{OTRAS CARACTERISTICAS}

Resistencia a los ácidos.

inalterable a la acción de los ácidos y vapores corrientes.

Combustibilidad . incombustible

Alcalinidad $\mathrm{pH}=7,5$

NOTA.-Los valores aqui facilitados se basan en una densidad media de $160,19 \mathrm{~kg} / \mathrm{m}^{3}$. La densidad del Foamglas varia de 144,17 a $176,21 \mathrm{~kg} / \mathrm{m}^{3}$. 


\section{PRODUCTOS Y APLICACIONES DEL FOAMGLAS}

En el proceso de fabricación del vidrio espumado se obtienen unos grandes bloques, que es preciso cortarlos convenientemente para obtener piezas de diferentes tamaños y formas, que sean manejables y cumplan adecuadamente la finalidad para la cual han sido concebidas. Estas piezas están convenientemente estudiadas para adaptarse a las necesidades constructivas; si es preciso retocarlas o cortarlas, para amoldarlas a una determínada dimensión o forma, puede realizarse tal operación valiéndose de las herramientas normales utilizadas en carpintería. Los bloques de Foamglas pueden cortarse con sierra, taladrarse con brocas corrientes y sobre ellos se pueden clavar y fijar puntas, clavos, etc. En las piezas de Foamglas, una vez cortadas a las dimensiones convenientes para formar las piezas normalizadas todas sus caras son superficies muy ásperas, constituídas por una multitud de celdillas abiertas. Estas superficies presentan una gran adherencia a toda clase de enlucidos, recubrimientos, morteros, másticos, etc. Esto supone una ventaja muy considerable desde el punto de vista constructivo, pues permite, con la ayuda de un medio adecuado, fijar estas piezas, entre sí o a otros materiales, con toda seguridad.

Para cubrir las distintas necesidades que se presentan al tener que resolver un problema de aislamiento, bien sea en un edificio, en una instalación industrial o en cualquier clase de construcción, se fabrican las siguientes piezas: placas, tiras de sección triangular, dovelas y coquillas.

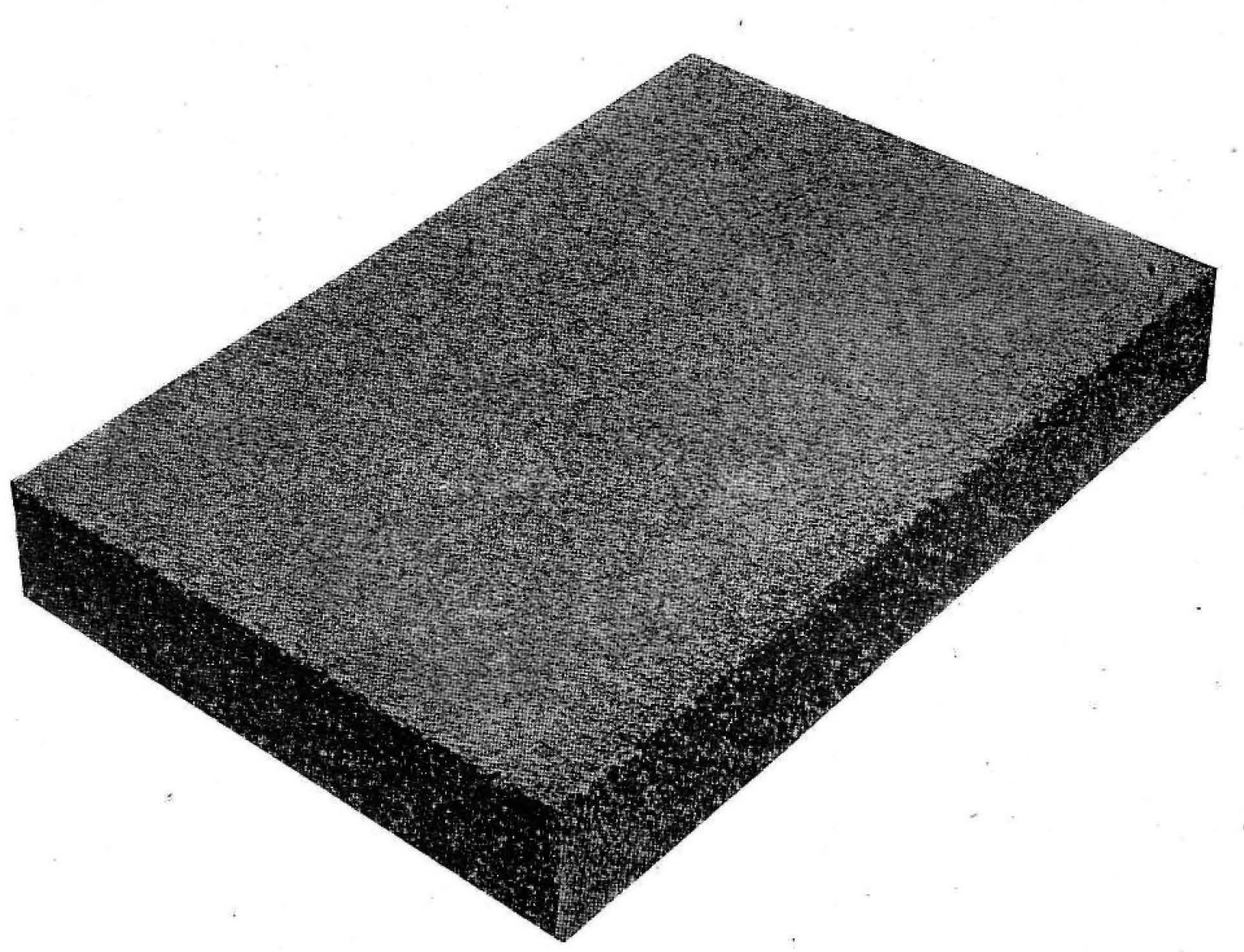

Fig. 1,-Placa de Foamglas 


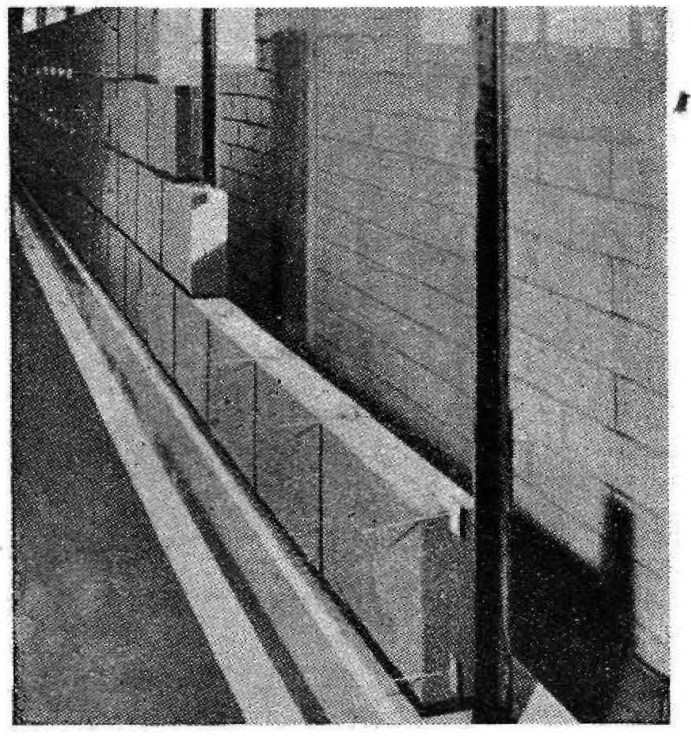

Fig. 2.-Construcción de tabiques

Fig. 3.-Revestimiento de muros

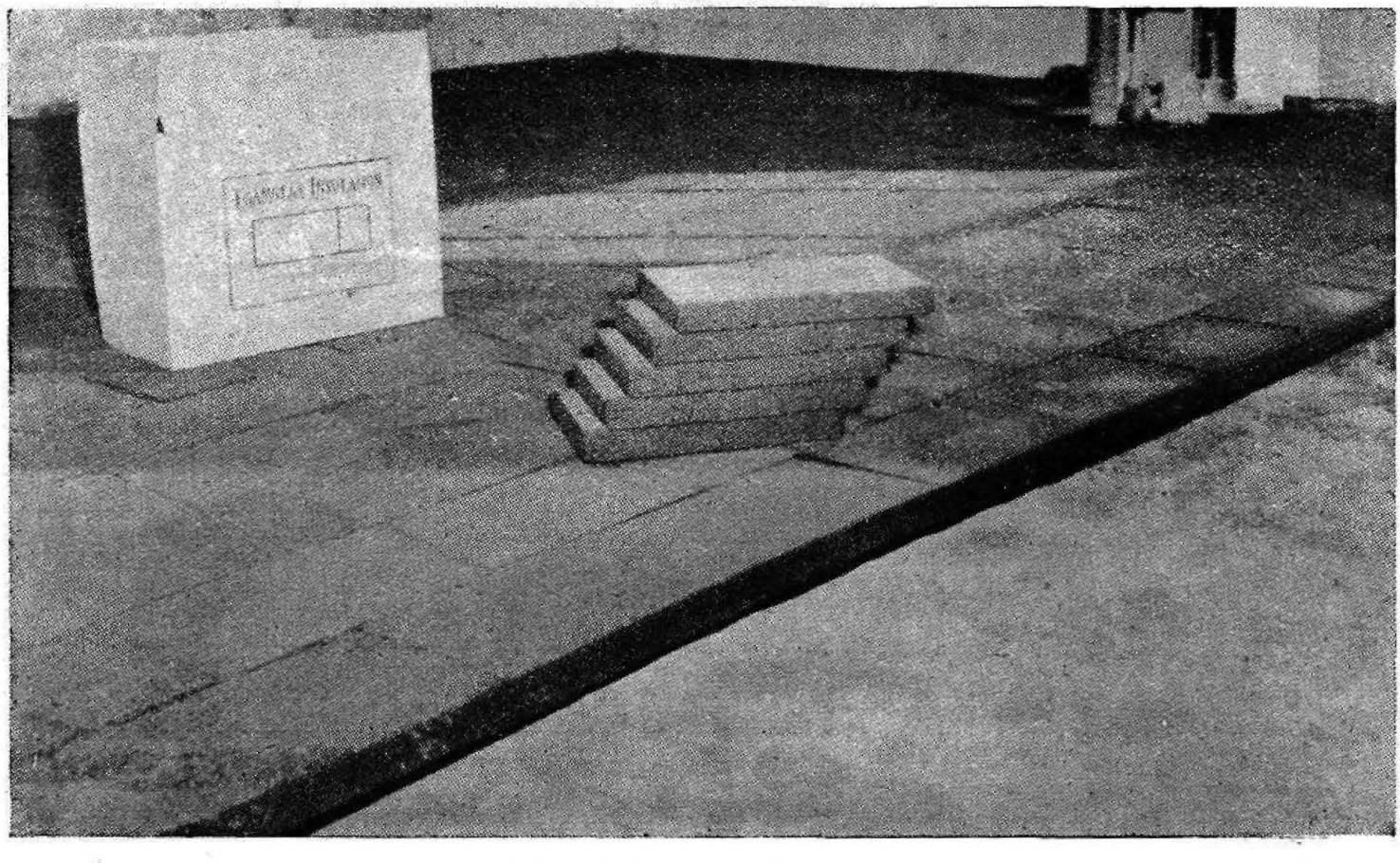

Fig. 4.-Aislamiento de suelos

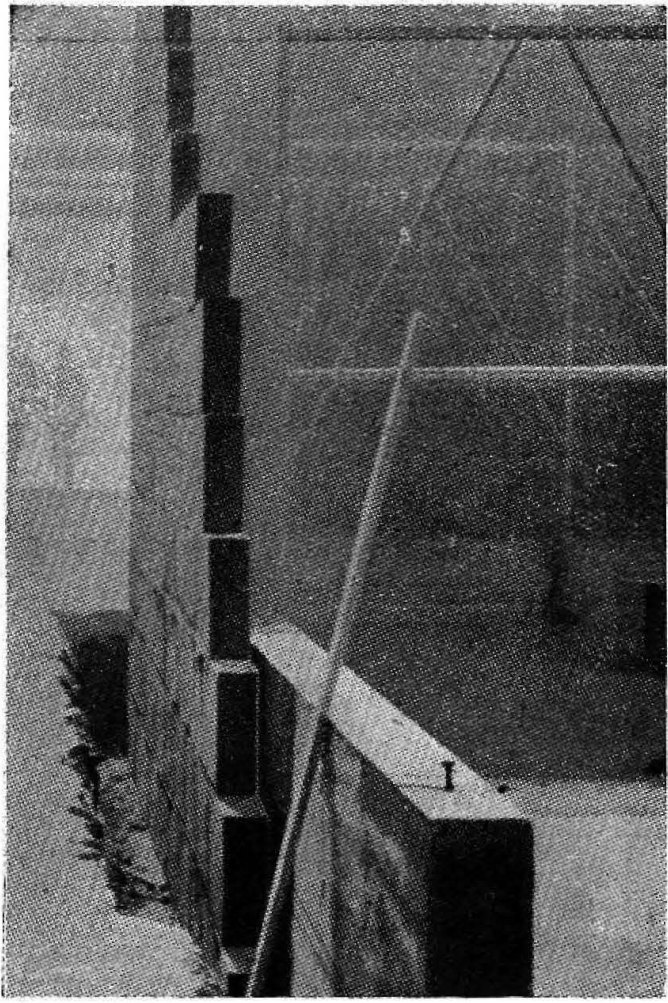




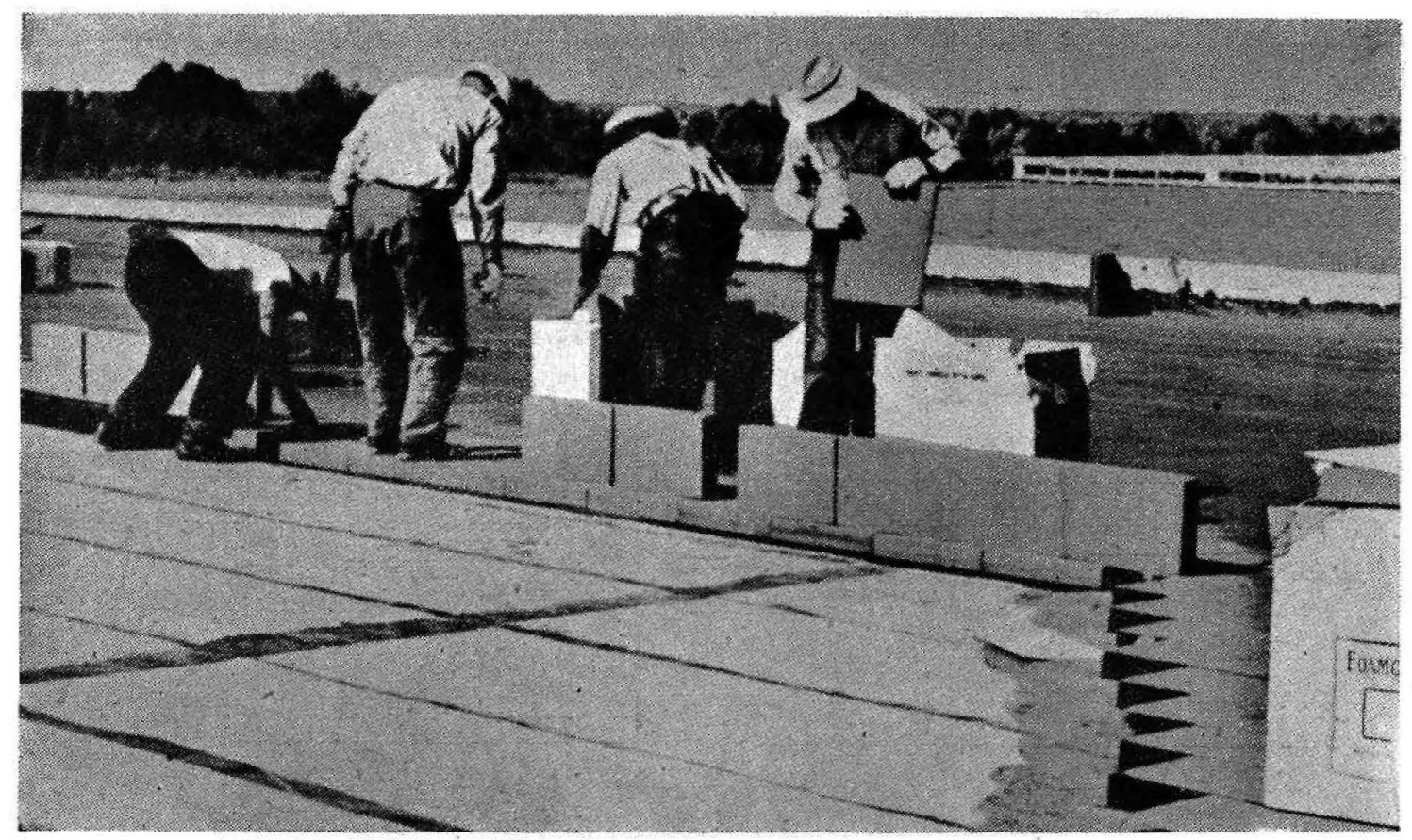

Fig. 5,-Aislamiento de cubiertas

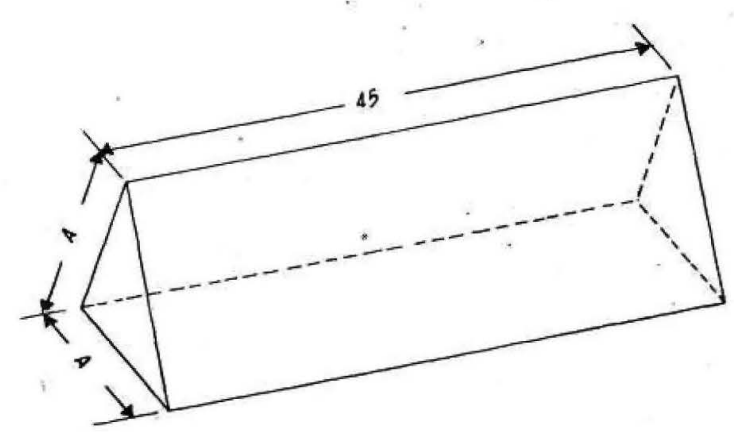

Fig. 6.-Pieza de sección triangular

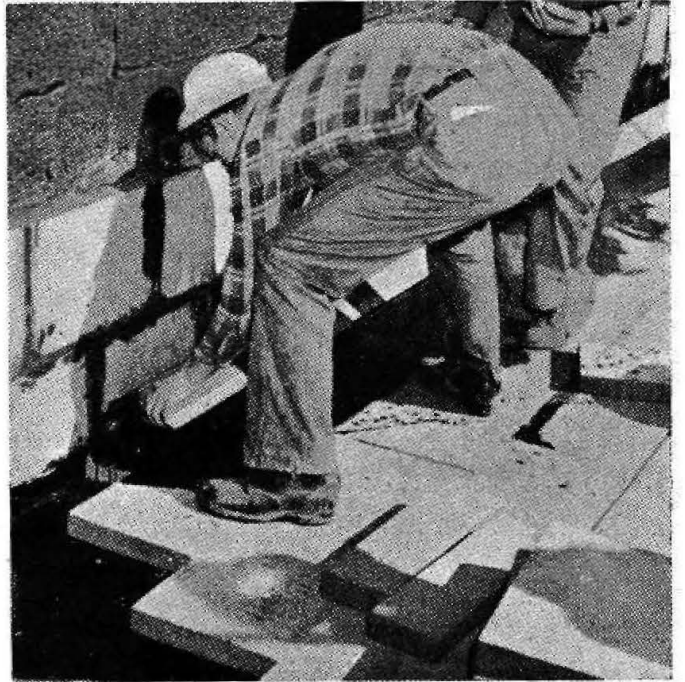

Fig. 7.-Aplicación típica de las piezas triangulares de FOAMGLAS en un encuentro de muro y suelo 


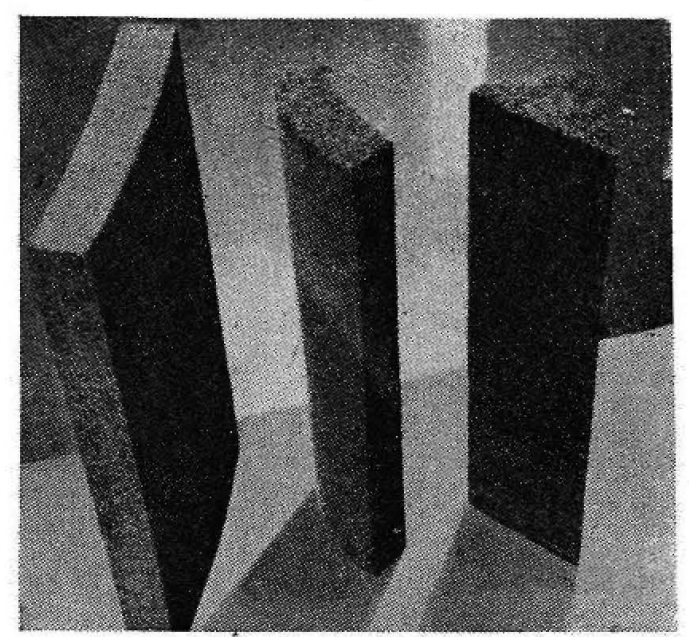

Fig. 8.-Piezas especiales aplantilladas

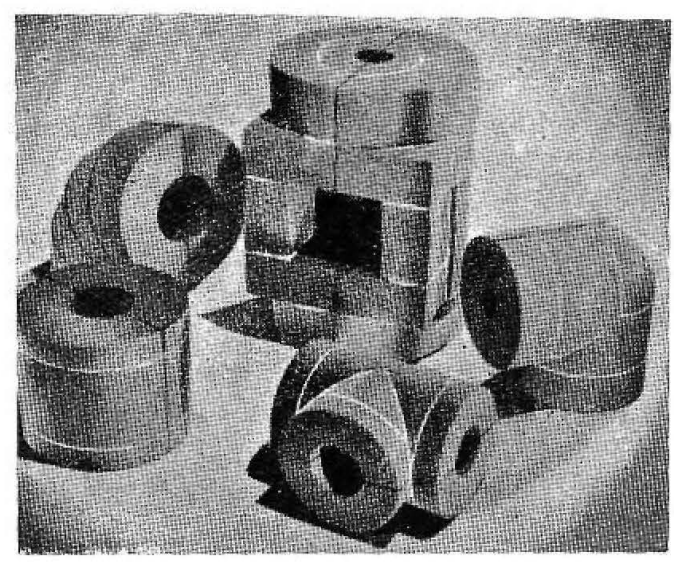

Fig. 10.-Piezas especiales para uniones, codos, ete.

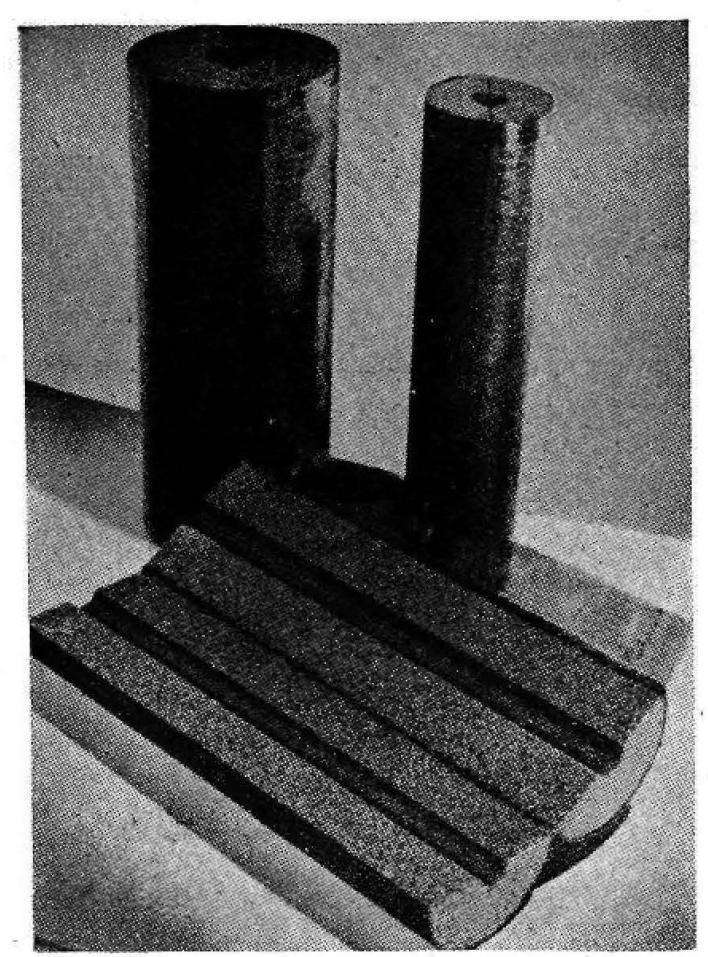

Fig. 9. - Coquillas de wFoamglas” para recubrimiento de tuberías 
Las placas de Foamglas (Fig. 1) sirven para la construcción de tabiques (Fig. 2), aislamiento de muros, suelos y techos (Figs. 3, 4 y 5). El tamaño de estas placas es de $30,5 \times 46 \mathrm{~cm}$ y los espesores son: $50,8,63,5,76,2,101,6$ y 127 milímetros.

Las tiras de sección triangular (Fig. 6) s̀e utilizan principalmente para suavizar el ángulo que forma una pared y el suelo o la cubierta de un edificio (Fig. 7). De esta forma, la membrana o el fieltro de impermeabilización no precisa ser doblado tan bruscamente, evitándose su agrietamiento La longitud de estas piezas es de $45 \mathrm{~cm}$ y la dimensión «A» puede ser de $10 \times 10$ ó $12 \times 12$ centímetros.

Además de las placas normales se fabrican piezas de forma especial (Fig. 8), que sirven para recubrir depósitos y chimeneas circulares.

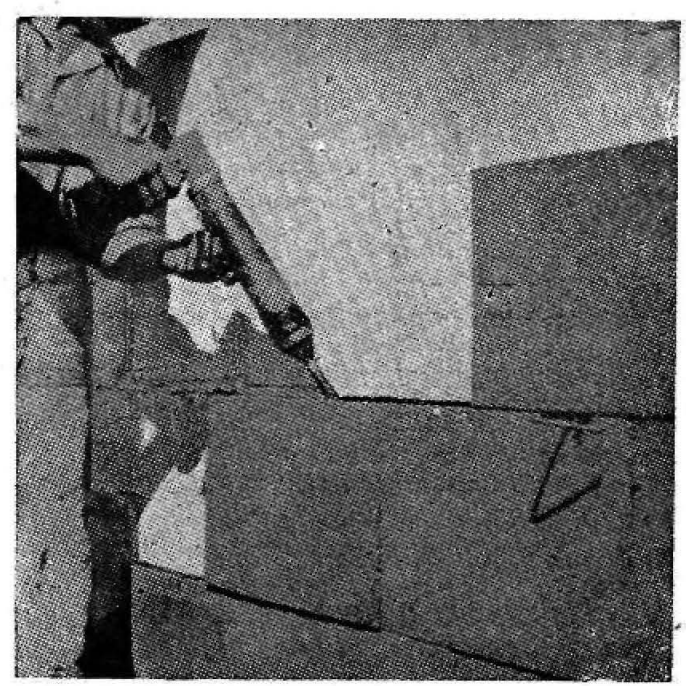

Fig. 11

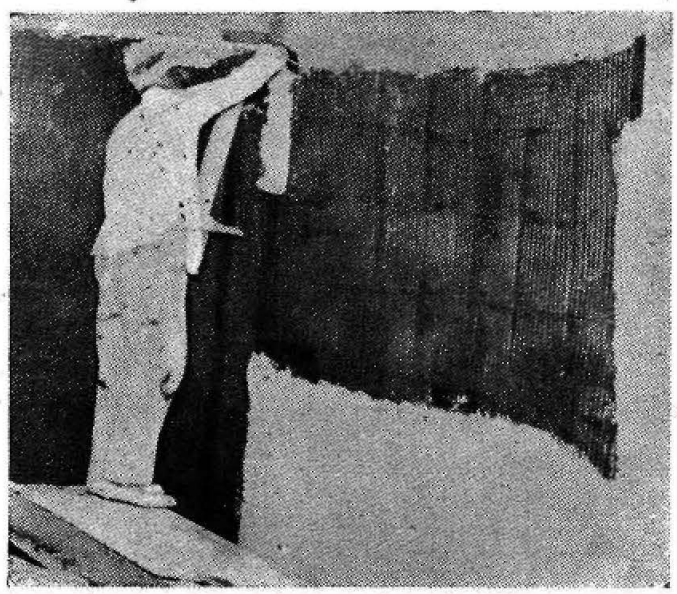

Fig. 12

Para aislar tuberías existen unas coquillas (Fig. 9), y piezas especiales (Fig. 10) para recubrir uniones, codos, etc.

La técnica de aplicación del Foamglas es sencilla. Existen numerosas soluciones constructivas, de acuerdo con el caso a resolver. En la abundante literatura de propaganda que facilita la Pittsburgh Corning Corporation existen unas amplias y detalladas recomendaciones para el empleo de este material. La mayor parte de las soluciones constructivas se realizan con la ayuda de materiales tradicionales. Sin embargo, hay que señalar el empleo de una mezcla de cera, amianto y asfalto, denominada «MASTIC 100», que sirve para impermeabilizar las juntas entre los bloques de Foamglas. Este mástico se aplica por medio de una bomba especial (Fig. II) y sirve como eficaz barrera contra la infiltración de vapores y para aquellos casos en que se precisa un adhesivo que no se seque y retraiga. Normalmente los bloques de Foamglas se fijan con adhesivos corrientes o con elementos de anclaje metálicos, de los cuales existen diversos tipos. Por último queda por señalar que el Foamglas puede recubrirse con los clásicos enlucidos de yeso o con cualquier mortero de cemento (Fig. 12), empleándose los sistemas ordinarios de aplicación. 